\title{
A necrotic cell death model in a protist
}

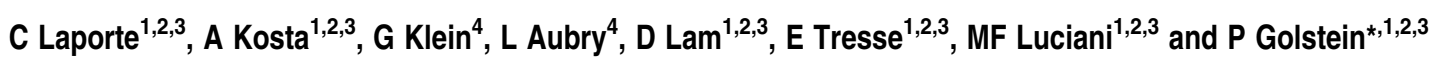

While necrotic cell death is attracting considerable interest, its molecular bases are still poorly understood. Investigations in simple biological models, taken for instance outside the animal kingdom, may benefit from less interference from other cell death mechanisms and from better experimental accessibility, while providing phylogenetic information. Can necrotic cell death occur outside the animal kingdom? In the protist Dictyostelium, developmental stimuli induced in an autophagy mutant a stereotyped sequence of events characteristic of necrotic cell death. This sequence included swift mitochondrial uncoupling with mitochondrial $2^{\prime}, 7^{\prime}$-dichlorofluorescein diacetate fluorescence, ATP depletion and increased oxygen consumption. This was followed by perinuclear clustering of dilated mitochondria. Rapid plasma membrane rupture then occurred, which was evidenced by time-lapse videos and quantified by FACS. Of additional interest, developmental stimuli and classical mitochondrial uncouplers triggered a similar sequence of events, and exogenous glucose delayed plasma membrane rupture in a nonglycolytic manner. The occurrence of necrotic cell death in the protist Dictyostelium (1) provides a very favorable model for further study of this type of cell death, and (2) strongly suggests that the mechanism underlying necrotic cell death was present in an ancestor common to the Amoebozoa protists and to animals and has been conserved in evolution.

Cell Death and Differentiation (2007) 14, 266-274. doi:10.1038/sj.cdd.4401994; published online 30 June 2006

Different types of cell death have long been defined in morphological terms. ${ }^{1,2}$ Thus, mammalian cells can die from 'apoptosis', 'necrosis' and 'autophagic cell death', and intermediate or mixed cell death types have also been reported. While caspase-dependent apoptosis has been studied in detail, less is known regarding the precise molecular mechanisms underlying caspase-independent types of cell death. These may play an important role especially in pathological circumstances and are thus now attracting considerable interest. Caspase-independent cell death mechanisms that are not prominent in the usual model organisms may be more experimentally accessible in simpler biological models, ${ }^{3}$ with potentially less interference from other cell death pathways. The underlying mechanisms may be phylogenetically conserved, which would be of interest in itself and would allow the extension of results obtained with simple models to higher eukaryotes.

In animal cells, necrosis is often defined as a nonapoptotic, nonautophagic type of cell death, with swelling of cells and organelles and, importantly, early plasma membrane rupture. ${ }^{4,5}$ At a molecular level, necrosis but not apoptosis is often accompanied by acute ATP depletion that is thought to cause cell death. ${ }^{6,7}$

Dictyostelium discoideum, a protist that emerged in evolution after plants and from an ancestor common to fungi and animals, ${ }^{8}$ shows developmental cell death. ${ }^{9}$ This can be mimicked in monolayers of Dictyostelium cells subjected to starvation and to the differentiation-inducing factor DIF-1 (hereafter designated $\mathrm{DIF}^{10}$ ). The type of cell death thus obtained includes vacuolization. ${ }^{11,12}$ Vacuolization is dependent on autophagy as inactivation of the atg1 autophagy gene ${ }^{13}$ prevented it. Importantly, when subjected to starvation and DIF, these atg1-1 cells still died, but now of a rapid nonvacuolar cell death. ${ }^{14}$

We report here that this nonvacuolar cell death in Dictyostelium possesses classical characteristics of necrotic cell death. This strongly suggests that the mechanism underlying necrotic cell death emerged in evolution before the divergence to Amoebozoa protists and animals.

\section{Results}

Rapid mitochondrial uncoupling. Hallmarks of necrotic cell death in mammalian cells include a rapid oxidative burst, mitochondrial uncoupling and plasma membrane rupture. To investigate whether early events following the addition of DIF to Dictyostelium HMX44A.atg1-1 cells ('cells', used throughout, unless mentioned otherwise) included an oxidative burst, we used the fluorescent probe $2^{\prime}, 7^{\prime}$ dichlorofluorescein diacetate (DC-FDA). Upon addition of DIF, starved cells stained fluorescent in the presence of DCFDA, as shown by microscopy and by flow cytometry (Figure 1). The same pattern of fluorescent labelling was obtained with dihydrorhodamine 123 and aminophenyl fluorescein, but not with hydroethidium or MitoSOX Red (not shown). Like other signs of nonvacuolar cell death, DCFDA fluorescence required prior starvation (not shown) and

${ }^{1}$ Centre d'Immunologie de Marseille-Luminy, Université de la Méditerranée, Case 906, Parc Scientifique de Luminy, 13288 Marseille Cedex 9, France; ${ }^{2}$ INSERM U631, 13288 Marseille Cedex 9, France; ${ }^{3}$ CNRS UMR6102, 13288 Marseille Cedex 9, France and ${ }^{4}$ CEA-Grenoble/DRDC/BBSI, UMR 5092 CNRS-CEA-UJF, 17, rue des Martyrs, 38054 Grenoble Cedex 09, France

${ }^{*}$ Corresponding author: P Golstein, Centre d'Immunologie de Marseille-Luminy, Case 906, Parc Scientifique de Luminy, 13288 Marseille Cedex 9 , France.

Tel: + 33-4-91-26-94-68; Fax: + 33-4-91-26-94-30; E-mail: golstein@ ciml.univ-mrs.fr

Keywords: cell death; necrosis; Dictyostelium; ATP; mitochondria; glucose

Abbreviations: CCCP, carbonyl cyanide 3-chlorophenylhydrazone; DC-FDA, 2',7'-dichlorofluorescein diacetate; DIF, differentiation-inducing factor; DNP, dinitrophenol; 2-DOG, 2-deoxyglucose; PI, propidium iodide; VDAC, voltage-dependent anion channel

Received 29.3.06; revised 21.4.06; accepted 11.5.06; Edited by JA Cidlowski; published online 30.6.06 

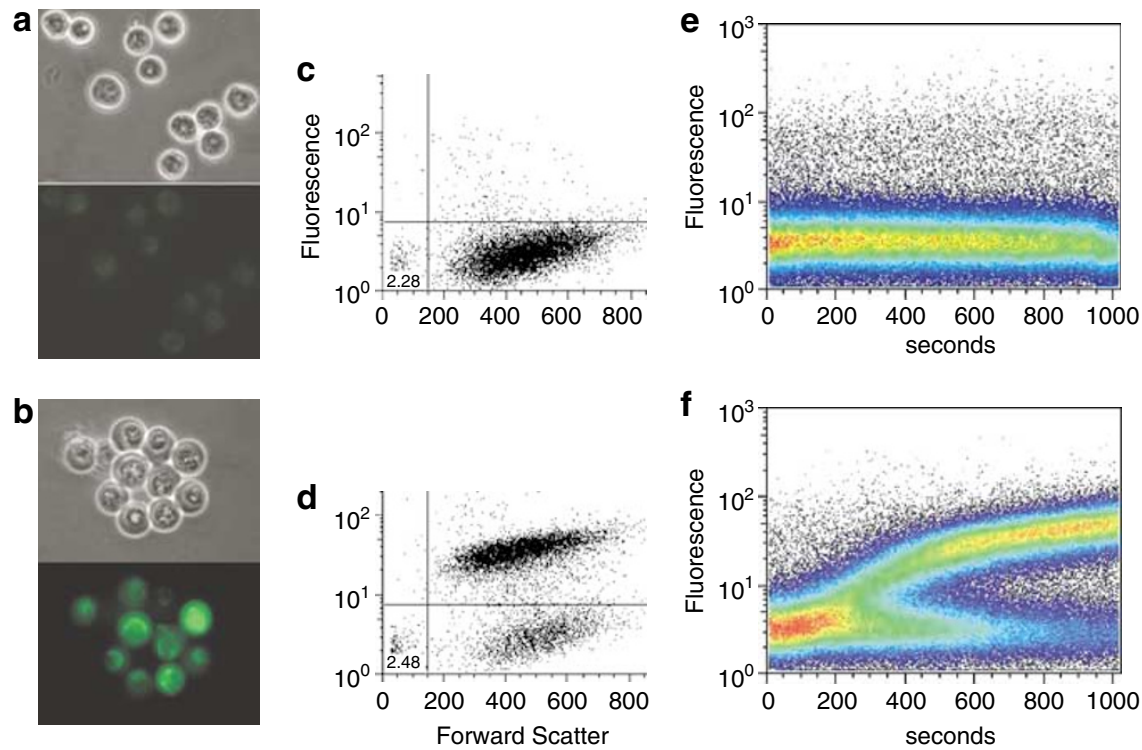

Figure 1 Induction of DC-FDA fluorescence following the addition of DIF. Starved HMX44A.atg1-1 cells were incubated with DC-FDA and with (b) or without (a) DIF for 15-30 min and analyzed by phase-contrast and fluorescence microscopy. The majority of cells were markedly fluorescent and showed perinuclear clustering of organelles only when DIF was added. Contrast and luminosity had to be increased to see the very weak fluorescence label of cells without DIF. Also, some photoactivation in DC-FDA-labelled cells occurred during fluorescence microscopy examination, leading to an increase in fluorescence only in cells that were already labelled, thus only among DIF-induced cells, therefore not affecting qualitative evaluation of labelling. (c, and d) Flow cytometry showed compared to cells without DIF (c) the presence of a 'cloud' of fluorescent cells upon addition of DIF (d). (e, and f) The emergence of fluorescent cells as a function of time was followed by continuous flow cytometry, starting the acquisition immediately after DIF addition. Cells without DIF remained negative (e), whereas after the addition of DIF some cells became fluorescent as soon as $200 \mathrm{~s}$, and most were fluorescent after $1000 \mathrm{~s}$ (f)

a

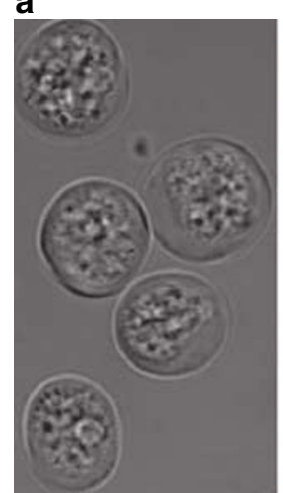

b

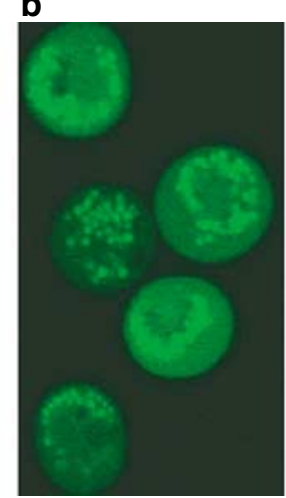

C

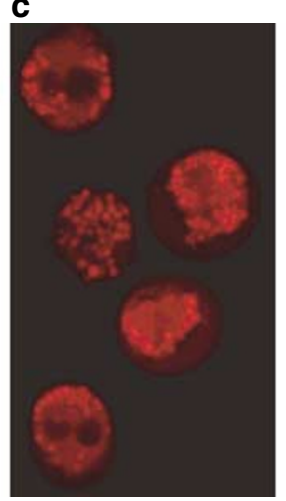

d

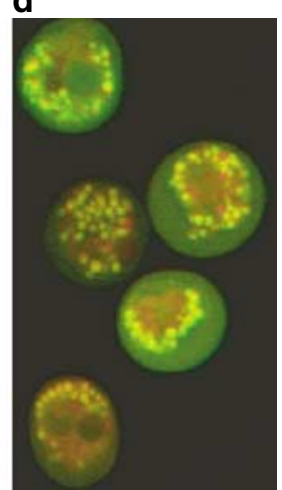

Figure 2 DC-FDA fluorescence is mitochondrial. Cells were observed about 30 min after the simultaneous addition of DIF, DC-FDA and MitoTracker Orange. (a) DIC microscopy, (b) DC-FDA fluorescence, (c) MitoTracker Orange fluorescence and (d) merge b and c

addition of DIF. Remarkably, as shown by flow cytometry some of the DC-FDA-exposed cells became fluorescent within 3 min after the addition of DIF and most of them were fluorescent after $15 \mathrm{~min}$ (Figure 1 and not shown). After a few minutes, only few cells with intermediate fluorescence levels could be detected (Figure 1d and f), indicating a rapid transition from no to full fluorescence in each cell. Confocal microscopy of cells after DIF addition showed for most stained structures colocalization of MitoTracker Orange (leading to red fluorescence of mitochondria) and DC-FDA (leading to green fluorescence), indicating that DC-FDA fluorescence was mitochondrial (Figure 2).

The fluorescence of DC-FDA and of dihydrorhodamine 123 is most often interpreted as revealing an oxidative burst, and it has been argued that oxidative stress can trigger necrotic cell death. However, we did not observe any inhibition of DC-FDA fluorescence and of membrane rupture by an extensive range of inhibitors of oxidative burst (not shown). This might be owing to limited membrane permeability in starved Dictyostelium cells (but see also for reservations as to interpretation of DC-FDA fluorescence ${ }^{15}$ ). This lack of effect of inhibitors prevented us from formally concluding on the existence of an oxidative burst early in this cell death pathway. In any case, this DC-FDA fluorescence revealed early DIF-induced events in mitochondria, presumably an oxidative burst, contemporary with mitochondrial uncoupling as shown below.

Unlike apoptotic cell death, mammalian necrotic cell death is often associated with ATP depletion.,6,7 In wild-type 
Dictyostelium cells, starvation led to some ATP depletion, which was not increased upon addition of DIF; this may be related to the absence of increased DC-FDA fluorescence in this case (not shown). In atg1-1 cells, starvation similarly led to ATP depletion. This apparent ATP depletion upon starvation in both parental and atg1-1 cells may merely result from a starvation-induced reduction in size occurring in both types of cells. However, in starved atg1-1 but not in wild-type cells, ATP per cell was further decreased upon addition of DIF (Figure 3a), likely through a decrease of the cellular concentration of ATP. Significant ATP depletion occurred within $3 \mathrm{~min}$ after the addition of DIF (Figure $3 \mathrm{~b}$ ). This ATP depletion suggested that DIF could directly or indirectly uncouple mitochondrial oxidative phosphorylation. An immediate consequence of such uncoupling should be an increase in oxygen consumption rate, which indeed occurred within less than a minute after the addition of DIF (or of dinitrophenol (DNP) as a positive control showing mitochondrial uncoupling) to starved cells (Figure $3 \mathrm{c}$ ). We conclude that addition of DIF to starved cells leads to almost immediate uncoupling of mitochondrial oxidative phosphorylation.

About 20 min after the addition of DIF, some cells became round with perinuclear clustering of cell organelles, which extended to almost all cells by $30 \mathrm{~min}$ (time-lapse videos in Online Supplementary Material). By electron microscopy, most cells showed at this time perinuclear clustering of otherwise dilated mitochondria. ${ }^{14}$ Clustering may be linked to the dependence of the actin system on energy supply by ATP. Perinuclear clustering has been reported also in circum- stances other than necrotic cell death, while dilation of mitochondria is usually associated with it.

Plasma membrane rupture. In animal cells, one of the most distinctive traits of necrotic cell death compared to other types of cell death is rapid rupture of the plasma membrane. In wild-type Dictyostelium cells, $50 \%$ of the cells showed membrane rupture about $36 \mathrm{~h}$ after the addition of DIF, as detected by propidium iodide (PI) uptake. ${ }^{11,12}$ In atg 1-1 cells, this occurred 30-300 min after the addition of DIF as shown by phase-contrast microscopy (Figure 4) and by time-lapse videos (Online Supplementary Material). Sequences of still frames from these videos allowed a more precise timing of the duration of the membrane rupture event itself. Only about $15 \mathrm{~s}$ were required to shift from an apparently intact plasma membrane to its almost complete disappearance (Figure 4a). In some but not all instances, an increase in apparent cell volume could be detected just before membrane rupture (time-lapse videos in Online Supplementary Material). This cell swelling and the mitochondrial swelling seen by electron microscopy ${ }^{14}$ are reminiscent of the classical cell and organelle swelling in mammalian necrotic cell death.

In all cases, plasma membrane rupture left behind some 'core' material (Figure 4a) whose aspect was distinct from that of the initial living cell. This physical change of dying cells suggested that detection and quantification of this cell death might be possible through measure of cell scatter. Indeed, flow cytometry showed upon addition of DIF the appearance of events of lower forward scatter (FSC) and slightly higher side scatter (SSC), generating a 'cloud' distinct from that of
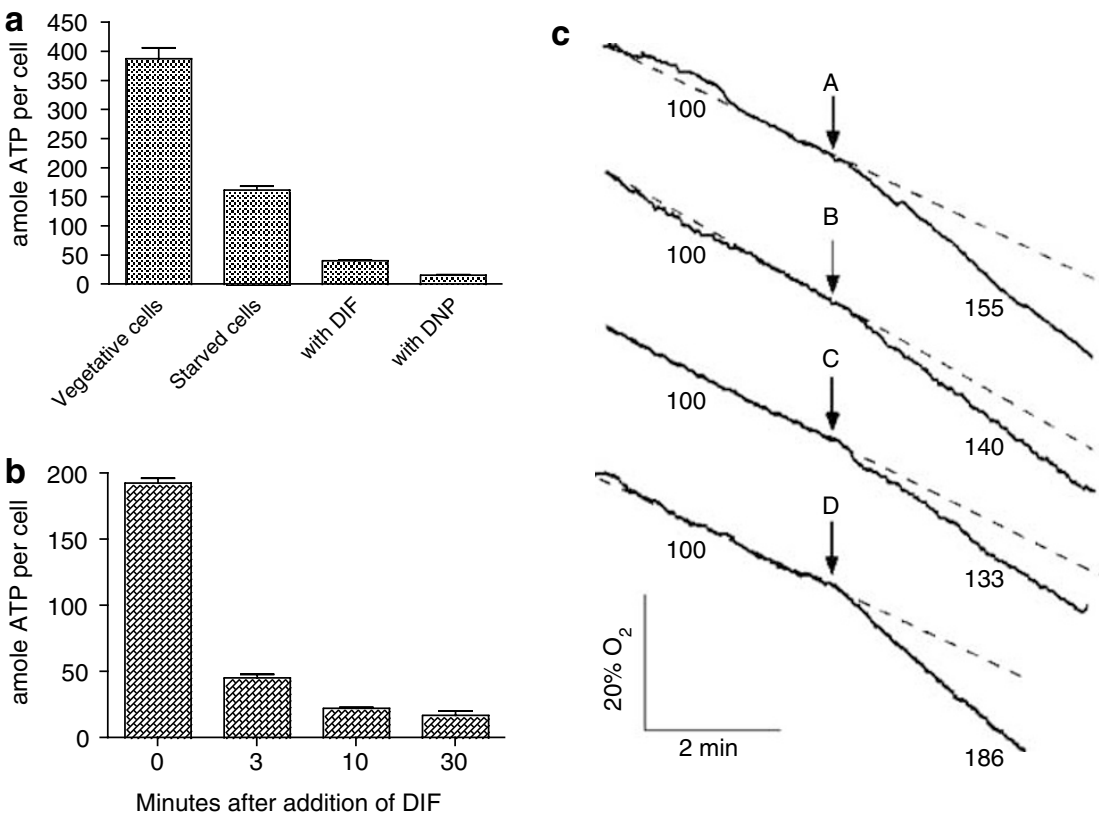

Figure 3 Mitochondrial uncoupling following the addition of DIF. (a) Stepwise ATP depletion in HMX44A.atg1-1 cells after starvation and incubation with DNP or DIF. Cells were either grown in HL5 medium (vegetative cells) or incubated overnight in MES buffer (starved cells). The latter cells were also incubated for 30 min with $100 \mathrm{nM}$ DIF or $100 \mu \mathrm{M}$ DNP. Perchloric cellular extracts were then prepared for determination of ATP levels per cell. Each column shows mean attomoles ATP per cell + S.E.M. of sextuplicate samples. (b) DIF induced most of the ATP depletion within 3 min as shown by the amount of ATP present in extracts from starved cells prepared $0,3,10$ or 30 min after DIF addition. (c) Increase in oxygen consumption rates upon addition of DNP or DIF. At the times indicated by arrows, DNP (A, $100 \mu \mathrm{M}$ ) or DIF (B, $100 \mathrm{nM}$; C, $250 \mathrm{nM}$; D, $500 \mathrm{nM}$ ) was added to starved atg1-1 cells, which led within less than a minute to an increase in oxygen consumption rate. Values below the traces indicate oxygen consumption rates as percentages of the rate before additions (indicated by the dashed line) 
a

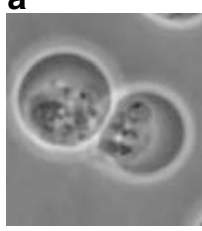

time 0

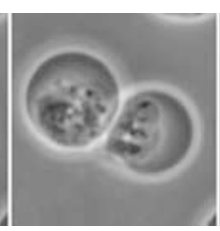

$10 \mathrm{sec}$

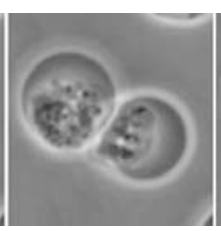

$15 \mathrm{sec}$

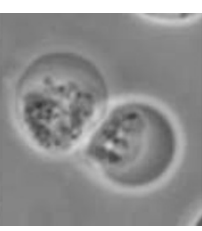

$20 \mathrm{sec}$

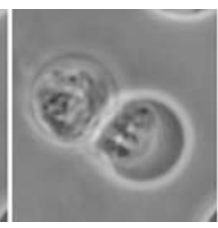

$25 \mathrm{sec}$

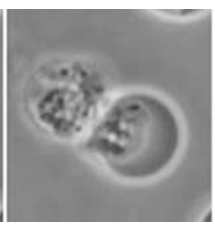

$30 \mathrm{sec}$

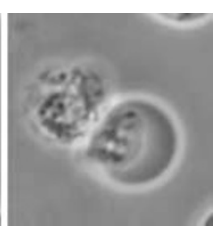

$35 \mathrm{sec}$
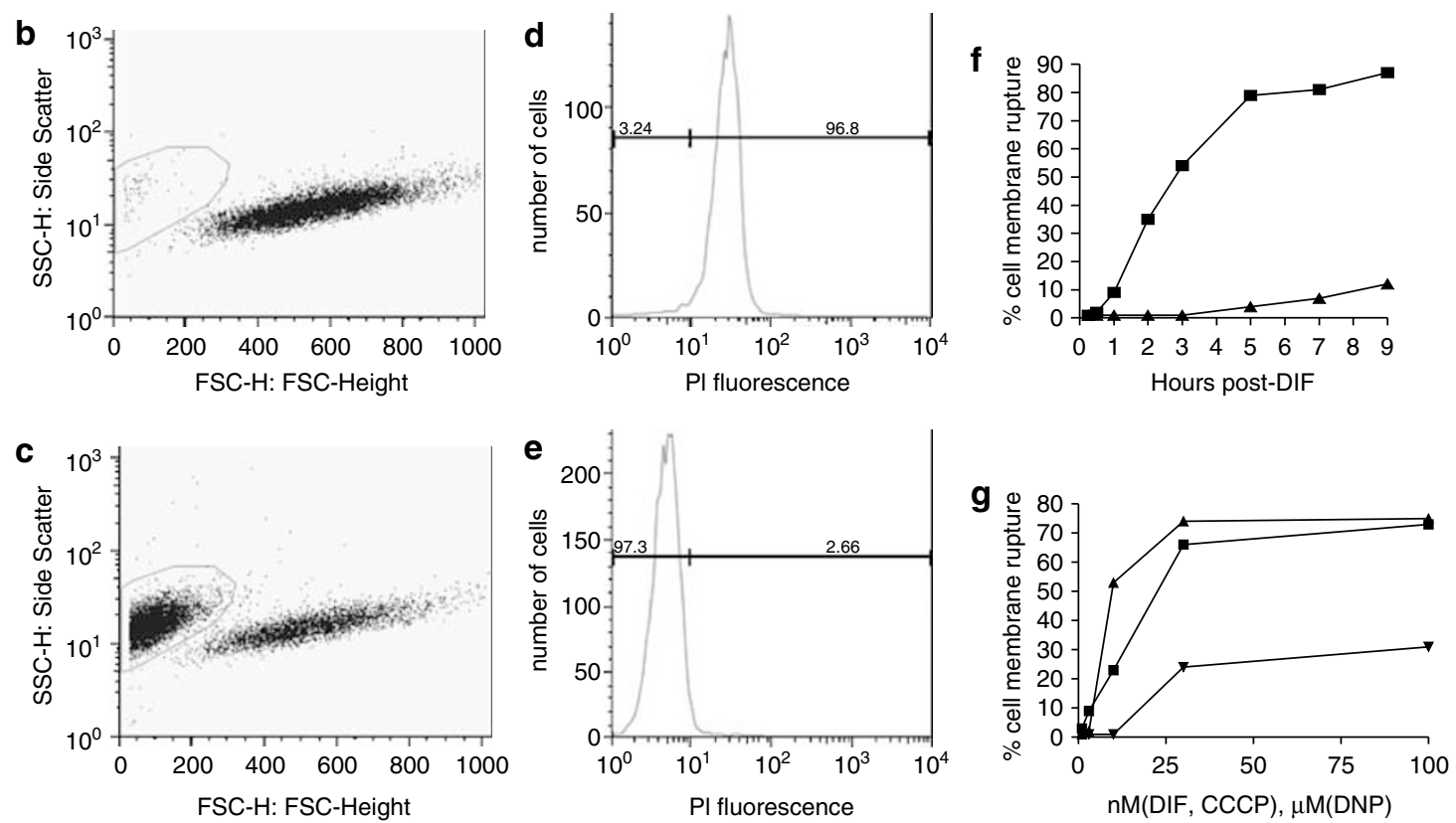

Figure 4 Membrane rupture following the addition of DIF or classical mitochondrial uncouplers. (a) Starved HMX44A.atg1-1 cells were subjected to time-lapse movies following the addition of DIF. A sequence of still frames extracted from time-lapse video 7 (in Online Supplementary Material) shows two cells $2 \mathrm{~h}$ after the addition of DIF, of which one shows rupture of its external membrane between the $20 \mathrm{~s}$ and the $35 \mathrm{~s}$ time points. (b and $\mathbf{c}$ ) Detection and quantification by FACS of cell membrane rupture. FACS output of forward versus SSC of HMX44A.atg1-1 cells, (b) without DIF or (c) $2.5 \mathrm{~h}$ after the addition of DIF. The latter shows a newly appeared 'cloud' of cells with ruptured membrane. (d and e) PI check of the proportion of dead cells in the cell clouds obtained by FACS (as in c). Before FACS analysis, cell populations were labelled with PI, which would only label cells with altered plasma membrane. (d) Cells from the cloud appearing after the addition of DIF were $97 \%$ positive, and (e) Cells from the pre-existing cloud were only $3 \%$ positive. (f) In another experiment, the percentage of cells in the newly appeared cloud in (c) that is, the percentage of cells dead according to the criterion of membrane rupture, could be followed with time after the addition of DIF; $\boldsymbol{\Delta}$, without DIF ; $\mathbf{\square}$, with DIF. (g) Percentage of starved cells with membrane rupture as a function of the concentrations of DIF $(\boldsymbol{\nabla}), \operatorname{DNP}(\mathbf{\Delta})$ or $\operatorname{CCCP}(\boldsymbol{\square}), 2.5 \mathrm{~h}$ after their addition, a time when the percentage cell membrane rupture for DIF-induced death, is not maximal and varies significantly from experiment to experiment. This percentage would rise further at later times, reaching a maximum around $5 \mathrm{~h}$ post-DIF (see $\mathbf{f}$ )

cells not subjected to DIF (Figure $4 \mathrm{~b}$ and $\mathrm{c}$ ). Cells in the cloud appearing after the addition of DIF were dead, as $96-98 \%$ were positive for PI staining compared to $2-4 \%$ in the preexisting cloud (Figure $4 \mathrm{~d}$ and e). Within the time frame of these experiments, no gross secondary degradation of dead cells occurred that might generate a high proportion of large debris counted as new dead cells, as judged by relative stability of dead cell 'cloud' parameters and by microscopy (not shown). As an additional check, in every experiment we systematically verified the state of cells by phase-contrast microscopy, and found a consistent and strong correlation between the degree of cell alteration seen at $60 \mathrm{~min}$ post-DIF and the \% cell membrane rupture measured by FACS at $2.5 \mathrm{~h}$ post-DIF.

Flow cytometry of unlabelled cells thus allowed quantitative assessment of the proportion of dead cells, for instance, at various times after the addition of DIF (Figure 4f). While without DIF cell death remained below $15 \%$ even at $9 \mathrm{~h}$, upon addition of DIF cell death reached a maximum of more than
$75 \%$ after $5 \mathrm{~h}$. Half-maximum values were reached at $2.5 \mathrm{~h}$ post-DIF, which was therefore used thereafter as a sensitive read-out time. The slope of the curve at this time indicated a speed of cell death of about $20 \%$ cells per hour (Figure $4 f$ ), a useful indication in time-lapse experiments that statistically one cell out of five would die in a given microscope field within $1 \mathrm{~h}$. In this report, we operationally define cell death as rupture of the cell membrane, resulting under the microscope in a smaller and immobile mass of mostly organelles or organelle remnants, which can be stained by $\mathrm{PI}$ and shows by flow cytometry a shift in scatter.

As shown above, DIF induces uncoupling of oxidative phosphorylation and plasma membrane rupture. Addition of the classical inducers of uncoupling DNP or carbonyl cyanide 3-chlorophenylhydrazone (CCCP) to starved (but not to unstarved) cells also led to ATP depletion and oxygen consumption increase, DC-FDA fluorescence, perinuclear clustering, and early plasma membrane rupture (Figures 3 and $4 \mathrm{~g}$ and not shown). Both latter traits were also observed in 
parental cells (not shown). This similar effect of dissimilar molecules all endowed with uncoupling activity indicated that in starved cells mitochondrial uncoupling may be sufficient for subsequent events including plasma membrane rupture. We then wished to investigate how this uncoupling can lead to plasma membrane rupture.

From mitochondrial uncoupling to membrane rupture, as probed with exogenous glucose. In mammalian cells, ATP depletion linked to uncoupling is often believed to be the cause of necrotic cell death, on the basis of results showing that provision of glucose led to both replenishment of ATP and prevention of necrotic cell death in mammalian cells. ${ }^{6,7,16-18}$ To try to link mitochondrial uncoupling and membrane rupture in Dictyostelium necrotic cell death, we investigated whether exogenous glucose would both prevent or delay cell death and replenish ATP, in this system in which the cells were deprived of glucose because of experimental starvation and atg1 mutation.

The addition of $5 \mathrm{mM}$ glucose indeed delayed DIF-induced cell death as assessed by membrane rupture (Figure 5a) by more than $6 \mathrm{~h}$, thus increasing more than fourfold the time required to reach a given level of cell death. The addition of glucose to atg1-1 cells (but not to parental cells, not shown) led to markedly less membrane rupture at $2.5 \mathrm{~h}$ after DIF in each of more than 30 experiments. The addition of glucose did not, however, prevent or grossly delay DC-FDA fluorescence (Figure 5b), showing that exogenous glucose affected a cell death stage downstream of mitochondrial events. DIFinduced glucose-delayed cell death was morphologically similar to that occurring earlier in the absence of glucose (not shown). Glucose delayed plasma membrane rupture induced not only by DIF but also by classical uncouplers (Figure 5c). The delay in membrane rupture observed a

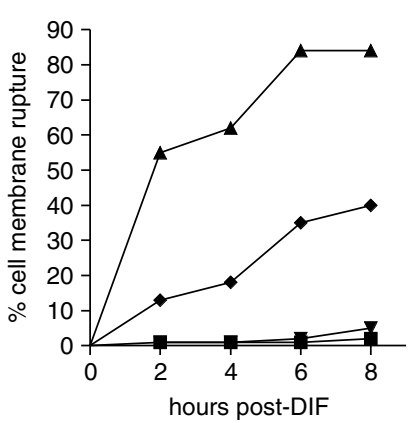

b

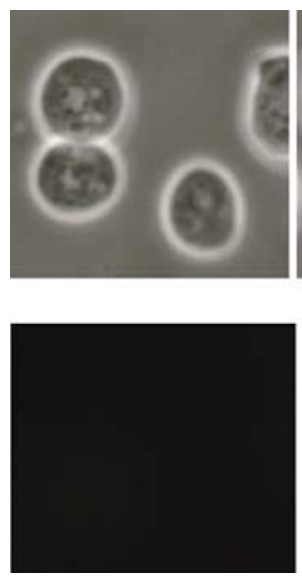

DIF
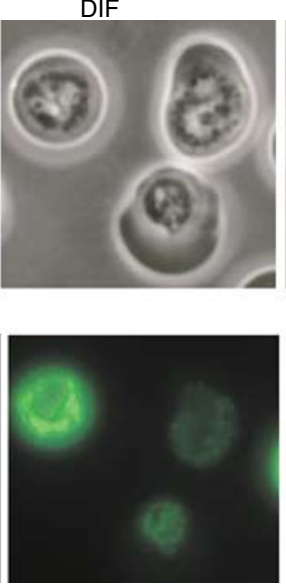

glucose
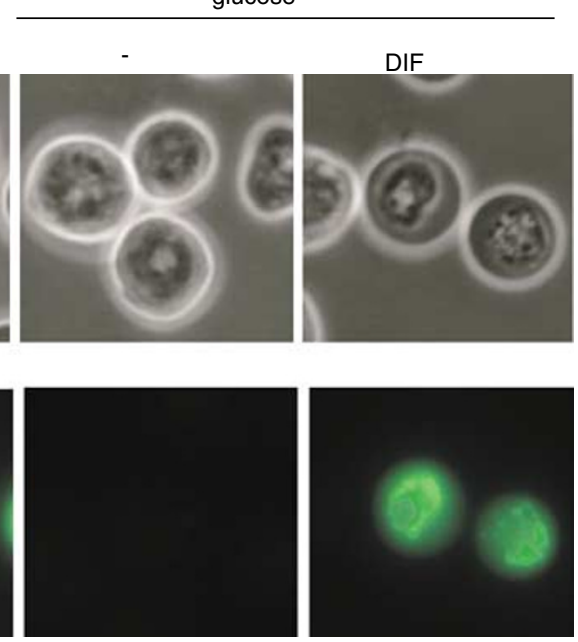

e

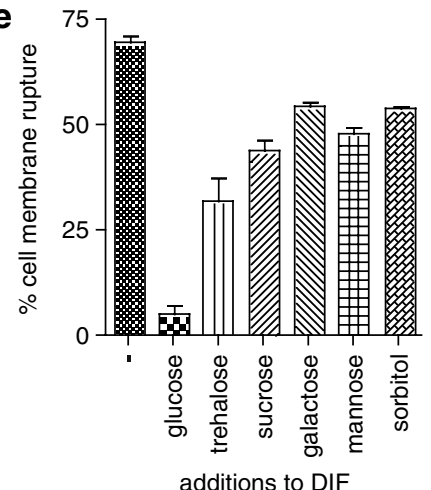

Figure 5 Glucose but not some other sugars delays cell membrane rupture. (a) Glucose slowed down DIF-induced cell membrane rupture. Starved HMX44A.atg1-1 cells were incubated with DIF in the absence $(\boldsymbol{\Lambda})$ or in the presence of $5 \mathrm{mM}$ glucose $(\bullet)$, and the percentage of cells with membrane rupture was followed with time. Controls without DIF with $(\mathbf{\square})$ or without glucose $(\mathbf{\nabla})$ showed almost no death. (b) Glucose did not grossly delay DIF-induced DC-FDA fluorescence or perinuclear clustering. Starved cells were incubated for $30 \mathrm{~min}$ in the presence of glucose $5 \mathrm{mM}$. Then, without discarding glucose, DC-FDA was added together with, or without DIF, and cells were checked by microscopy after another $30 \mathrm{~min}$. All fluorescent pictures were taken with a set exposure time of $2 \mathrm{~s}$. In each labelled cell, organelles form a fluorescent cluster around a much smaller nucleus. (c) Also uncoupler-induced cell membrane rupture is delayed by glucose or 2-DOG but not by sorbitol. Starved cells were preincubated for 30 min with no sugar, or $5 \mathrm{mM}$ of glucose, 2-DOG or sorbitol (from left to right in each group of four columns, respectively), then in some chambers $100 \mathrm{nM}$ of DIF, or $30 \mu \mathrm{M}$ of DNP, or $30 \mathrm{nM}$ of CCCP, or no uncoupler, was added as indicated. Cell membrane rupture was checked $2.5 \mathrm{~h}$ after this addition. (d) Glucose delays cell membrane rupture more than fructose or sorbitol. Starved cells were incubated for $30 \mathrm{~min}$ in the absence or in the presence of $1-6 \mathrm{mM}$ of glucose $(\boldsymbol{\nabla})$, fructose $(\mathbf{\Delta})$ or sorbitol $(\boldsymbol{\nabla})$, then without removing the sugars DIF was added. Percentage cell membrane rupture was measured by flow cytometry after $2.5 \mathrm{~h}$ of incubation. (e) Glucose delays cell membrane rupture more than each of a range of sugars and than sorbitol. Starved cells were incubated for $30 \mathrm{~min}$ in the absence or in the presence of $5 \mathrm{mM}$ of the indicated sugar, and treated as in (d) 
with exogenous glucose was not, or far less, observed with $5 \mathrm{mM}$ sorbitol or fructose (Figure $5 \mathrm{~d}$ ), thus excluding a simple osmotic effect. Also, trehalose and to a lesser extent sucrose, known to confer more efficiently than glucose cell resistance to an abiotic stress possibly through peculiar solution properties (for a review see Crowe et al. ${ }^{19}$ ), were far less efficient than glucose at delaying membrane rupture (Figure 5e), making this explanation unlikely in the present situation.

Although glucose delayed DIF-induced plasma membrane rupture, in marked contrast addition of galactose or mannose led to no more membrane rupture delay than the sorbitol control (Figure 5e). This showed that specific recognition of glucose was required for its membrane effect. 6-Deoxyglucose led to little membrane rupture delay (Figure 6a), showing that $\mathrm{C} 6$ is significant for the delaying effect of glucose. Importantly, 2-deoxyglucose (2-DOG), that can be C6phosphorylated but cannot undergo glycolysis past this phosphorylation, led to a delay in DIF (and also DNP and $\mathrm{CCCP}$ )-induced membrane rupture (Figures $5 \mathrm{c}$ and $6 \mathrm{a}, \mathrm{c}$ ), however sometimes less marked than with glucose. This result showed that at least part of the membrane-rupturedelaying effect of glucose can occur without glycolysis. Consistent with the 2-DOG results, addition of glucose $5 \mathrm{mM}$ did not obviously replenish ATP (Figure 6b) tested 5-120 min after addition of DIF (not shown). In contrast, addition as a control of cell-permeant methylpyruvate replenished ATP or prevented its depletion (Figure $6 \mathrm{~b}$ ) and also prevented DIFinduced DC-FDA fluorescence, perinuclear clustering (not shown) and membrane rupture (Figure 6c). Altogether, in Dictyostelium necrotic cell death exogenous glucose delayed membrane rupture otherwise than through glycolysis and ATP replenishment, strongly indicating a nonglycolytic effect of glucose in this case.

\section{Discussion}

Necrotic cell death: from mitochondrial uncoupling to cell membrane rupture. Following addition of DIF to starved atg1-1Dictyostelium cells, early events occurring within 1-3 min include DC-FDA mostly mitochondrial fluorescence, ATP depletion and oxygen consumption increase, all of these pointing toward mitochondrial uncoupling. This is consistent with the fact that DIF can be replaced by the classical uncouplers DNP or CCCP. These results are complementary to those of Shaulsky and Loomis, ${ }^{20}$ who showed that DIF was able to reduce the membrane potential of isolated submitochondrial particles. Both sets of results lead to the conclusion that there is an uncoupling effect of DIF. The results of Shaulsky and Loomis $^{20}$ indicated that the uncoupling effect of DIF may occur through its direct interaction with mitochondria. Thus, remarkably the DIF differentiation factor may act at least in part through rapid uncoupling of oxidative phosphorylation.

In Dictyostelium development, DIF has also been shown to activate transcription factors. When added after a $4 \mathrm{~h}$ starvation period, DIF led within $2-3 \mathrm{~min}$ to the tyrosine phosphorylation of the STATc transcription factor, ${ }^{21}$ thus detectably preventing the exit of STATc from the nucleus, ${ }^{22}$ and induced changes in subcellular localization of the transcription factor DimB. ${ }^{23,24}$ The relationship between DIF-induced rapid phosphorylation events and DIF-induced rapid uncoupling of oxidative phosphorylation is not known.

The fact that distinct moieties such as DNP and CCCP (classical uncouplers) and DIF induced both mitochondrial uncoupling and plasma membrane rupture strongly indicated that in this system mitochondrial uncoupling is sufficient for membrane rupture. Whether there is a causal relationship between ATP depletion and membrane rupture could not be a

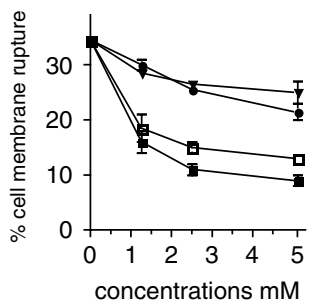

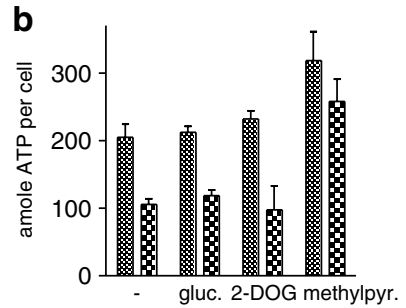

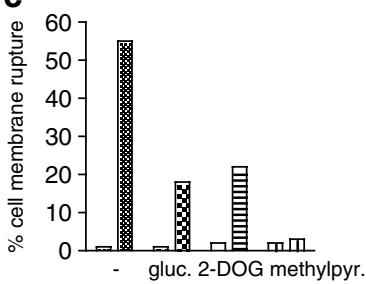

30-300 $\min$

Plasma

membrane

rupture

starvation DC-FDA fluor.

Oxygen cons.

glucose

Figure 6 Further characteristics of glucose-mediated delay of membrane rupture. (a) Effect of glucose ( $\mathbf{\square})$, sorbitol $(\boldsymbol{\nabla})$, 2-DOG (open square) or 6-deoxyglucose (filled circle) on cell membrane rupture. Cells were treated as in Figure $5 \mathrm{~d}$. (b) Glucose or 2-DOG do not replenish ATP, whereas methylpyruvate prevents ATP depletion. Starved cells were preincubated for $30 \mathrm{~min}$ with no sugar, or $5 \mathrm{mM}$ of glucose, 2-DOG or methylpyruvate as indicated, then in some chambers DIF was added (right column of each group of two). The amount of ATP per cell was measured $30 \mathrm{~min}$ after the addition of DIF. (c) Glucose but also 2-DOG and methylpyruvate delay cell membrane rupture. Starved cells were incubated for $30 \mathrm{~min}$ in the absence or in the presence of $5 \mathrm{mM}$ glucose, 2-DOG or methylpyruvate as indicated, then DIF was added in some chambers (right column in each group of two). Percentage cell membrane rupture was measured by flow cytometry after $2.5 \mathrm{~h}$ of incubation. (d) Schematic representation of the Dictyostelium necrotic cell death model. Starved atg 1-1 cells to which the differentiation factor DIF is added undergo mitochondrial uncoupling. This is sufficient for subsequent plasma membrane rupture as shown by the effect of classical uncouplers. The addition of exogenous glucose delays plasma membrane rupture in a nonglycolytic manner 
investigated in this system using glucose, because glucose affected membrane rupture otherwise than by replenishing ATP. Thus, mitochondrial uncoupling seems sufficient to ensure plasma membrane rupture, but it is not known whether this is through ATP depletion (although ATP depletion is a convenient explanation for alterations of the cytoskeleton leading to perinuclear clustering of mitochondria, and of membrane ATP-dependent molecular pumps contributing to membrane rupture).

Plasma membrane rupture delayed by glucose. In mammalian necrotic cell death, glucose-induced rescue was explained through concomitant ATP replenishment. ${ }^{6,7}$ In Dictyostelium necrotic cell death, addition of glucose or of 2-DOG led to membrane rupture delay with no ATP replenishment (this report). At least in Dictyostelium, factors other than ATP depletion govern membrane rupture in necrotic cell death, and can be controlled by exogenous glucose. In mammalian necrotic cell death, these factors may occur, but may have been ignored owing to the readily available explanation of ATP replenishment through glycolysis.

Exogenous glucose may act extracellularly to stabilize the membrane, either by a direct effect or through transmembrane signaling by unspecified glucose receptors. Exogenous glucose may also, or instead, act intracellularly, which we tend to favor based on the requirement for C6 phosphorylation and on the commonly accepted intracellular location of hexokinases/glucokinases. Once in cells, to delay membrane rupture glucose must be both specifically recognized and C6phosphorylated, presumably by a glucokinase. Dictyostelium possesses no hexokinases but structurally unrelated glucokinases (Eichinger et al. ${ }^{25}$; see also http://dictybase.org/), which, of note, showed activity in cells undergoing development. ${ }^{26}$ Glucose delayed plasma membrane rupture but not DC-FDA fluorescence, perinuclear clustering of mitochondria or ATP depletion. Thus, the delaying effect of glucose was downstream or independent of early mitochondrial events.

The efficacy of 2-DOG and the absence of ATP replenishment strongly indicate that the effect of glucose on membrane rupture was not through glycolysis. In animal cells, glucose can modulate hexokinases in an energy-independent manner leading to voltage-dependent anion channel control of mitochondria, thereby affecting apoptotic cell death. ${ }^{27-30}$ Also, in some animal cells glucose inhibited cell death apparently through the pentose phosphate pathway. ${ }^{31}$ As an example, some mammalian cells could be protected from $\mathrm{H}_{2} \mathrm{O}_{2}$-induced necrotic cell death by 2-DOG flowing into the pentose phosphate pathway and producing NADPH; exogenous glucose led only or mostly to glycolysis, and the pentose phosphate pathway was favored only when 2-DOG was added instead of glucose. ${ }^{32}$ In starved Dictyostelium cells, for unknown reasons exogenous glucose cannot undergo glycolysis. In both cases, (Le Goffe et al. ${ }^{32}$, this report), ATP was not produced.

Altogether, we conclude that mitochondrial uncoupling leads to plasma membrane rupture in this necrotic cell death, whereas exogenous glucose delays it nonglycolytically (Figure 6d).
Dictyostelium necrotic cell death and the phylogenesis of cell death. Dictyostelium, a protist, belongs to the unikont group of eukaryotes together with fungi and animals. ${ }^{33}$ The present results show that a similar necrotic cell death pathway can operate in both animal cells and Dictyostelium. Necrosis would thus seem more widespread and more ancient than apoptosis dependent on bona fide caspases, which is restricted to the animal kingdom. Necrosis must have existed in an ancestor common to Dictyostelium and animals, and may thus qualify as a primordial cell death mechanism within unikont eukaryotes. It will be of interest to check whether a similar necrotic cell death mechanism exists among eukaryotes in other unikonts such as fungi, and in bikonts such as plants.

The existence of several distinct types of cell death coexisting in the same cell (for a review see Golstein and Kroemer ${ }^{34}$ ) raises the question of which selective advantage led to their emergence in evolution. This bears on phylogenetic evolution of a function, as opposed to that of a molecule or of a species. In phylogeny, a primordial (necrotic) cell death mechanism may have been conserved through at least some eukaryotic kingdoms. This conservation of necrotic cell death may have been accompanied by serial addition of molecules allowing other types of cell death, for instance, caspases at the emergence of the animal kingdom to allow apoptosis, perhaps initially to facilitate cell corpse dismantling. ${ }^{34}$

More specifically, in a remote ancestor of Dictyostelium, a primordial necrotic mechanism of cell death may have operated upon starvation, possibly on some isolated cells to ensure survival of others. Addition upon evolution of atg1 and autophagy would have allowed initial developmental aggregation (which atg1-1 mutant cells cannot undergo ${ }^{35}$ ). It would have led also to a much slower course of cell death in Dictyostelium (compare the kinetics of death for atg1-1cells in this report and for wild-type cells ${ }^{11}$ ). A slower course of cell death during Dictyostelium development would have provided enough time in this development for morphogenetic signalling of prestalk to prespore cells, cellulose synthesis and formation of a stalk. This may be an example of evolutionary modulation of cell death (interestingly, modulation of mechanism rather than of occurrence) inducing/allowing a shift in developmental pattern.

A model for the study of necrotic cell death. Dictyostelium stalk cell death, at least as mimicked in monolayer cultures, is triggered by DIF and starvation, which are physiological stimuli of Dictyostelium development. This cell death is autophagic vacuolar for parental cells and necrotic for atg1-1cells. Thus, Dictyostelium cell death (Kosta et al. $^{14}$; this report) is necrotic when autophagy is prevented, reminiscent of mouse embryonic interdigital cell death being necrotic when apoptosis is prevented, ${ }^{36}$ providing examples of necrotic cell death resulting from developmental signals at least when other cell death mechanisms are inhibited.

Necrotic death of atg1-1 Dictyostelium cells is one of the very few instances where a developmental trigger leads in a cell line to death in the absence of genes encoding bcl-2 family members, or caspase or metacaspase genes (its only paracaspase gene is not involved in cell death ${ }^{37}$ and Lam 
et al., in preparation). This cell line can undergo neither autophagy nor apoptosis, thus ensuring minimal interference from other types of cell death with its necrotic cell death. Dictyostelium bears a haploid and now sequenced genome, which contributes to its experimental and genetic tractability. Together with a few other thoroughly studied models (for instance, mammalian cells $\mathrm{s}^{4,38}$ and Caenorhabditis elegans ${ }^{5}$ ), this model is thus attractive for further investigation on molecular mechanisms of necrotic cell death.

\section{Materials and Methods}

Cells, cell culture and light microscopy. $D$. discoideum axenic strains HMX44A (for derivation, see Levraud et al. ${ }^{12}$ ) and HMX44A. atg1-1 (an HMX44A derivative mutated for the autophagy gene atg $1^{14}$ ) were routinely grown at $22^{\circ} \mathrm{C}$ in $\mathrm{HL} 5$ medium $^{39}$ as modified, ${ }^{11}$ except for maltose which was $9 \mathrm{~g} / \mathrm{l}$. Blasticidin $(10 \mu \mathrm{g} /$ $\mathrm{ml}$ ) was added to the HMX44A.atg1-1 cultures.

For experiments, unless stated otherwise, vegetative cells in growth phase were washed once and resuspended in $10 \mathrm{mM}$ MES (MES hydrate, Sigma M2933) prepared in demineralized water. The $\mathrm{pH}$ of this MES buffer was found to matter The $\mathrm{pH}<6$ increased, and $\mathrm{pH}>6.6$ decreased cell membrane rupture, perinuclear clustering and DC-FDA fluorescence (not shown). The $\mathrm{pH}$ was therefore adjusted at 6.4 with $\mathrm{NaOH}$ in all experiments, and every molecular species tested in this report was checked not to alter this $\mathrm{pH}$ by more than $0.2 \mathrm{pH}$ Units. The cells were adjusted at a concentration of $3 \times 10^{5} \mathrm{cell} / \mathrm{s} / \mathrm{ml}$. One milliliter of this cell suspension was distributed in each well of Lab-Tek culture chambers (ref. 155380, Nalge Nunc, Naperville, IL, USA; these have a thin glass coverslip bottom allowing high-power inverted fluorescence microscopy) with the addition of cAMP to a final concentration of $3 \mathrm{mM}$. Incubation proceeded for $16 \mathrm{~h}$ at $22^{\circ} \mathrm{C}$ in MES buffer and cAMP. The previously used $8 \mathrm{~h}$ incubation period ${ }^{11,40}$ was found limiting for atg1-1cells. A starvation period of sufficient duration was essential for efficient induction by DIF of necrotic cell death. Cells were then washed once by careful removal and addition of MES and incubated at $22^{\circ} \mathrm{C}$ in either MES, or MES in the presence of the differentiation factor DIF-1 (DN1000, Affiniti Research Products, Exeter, UK) at a final concentration of $10^{-7} \mathrm{M}$, unless stated otherwise (varying the concentrations of DIF did not modify the type of death but changed it quantitatively: when tested at $7 \mathrm{~h}$ post-DIF, percentage cell membrane rupture and perinuclear clustering induced by $10^{-7} \mathrm{M}$ DIF dropped by about $50 \%$ at $3 \times 10^{-8} \mathrm{M}$, and was almost not detectable at $10^{-8} \mathrm{M}$ ). After incubation for the indicated period of time, cells in the Lab-Tek chambers were examined using an inverted Zeiss Axiovert 200 microscope usually with $a \times 100$ oil-immersion objective. Pictures were taken using an Axiocam MRC digital camera connected to a PC equipped with Axiovision (Zeiss). In some cases, pictures were taken using a confocal microscope (Zeiss Axiovert 200 LSM510) with a $\times 63$ oil immersion objective, with the scanning module at $1024 \times 1024$ pixel resolution, 8 bits, using a scan average of 4 , and Argon/2 and HeNe1 lasers with BP 505-530 and LP 560 filters. Images were analyzed with LSM software using false colors. Images and time-lapse videos were transferred to a Macintosh computer and treated with Graphic Converter. Figure assemblies were carried out using FreeHand and Illustrator.

Measurement of cell membrane rupture. Flow cytometry enables to quantify the proportion of cells with a disrupted membrane. Cells in Lab-Tek chambers were resuspended and transferred to tubes and data acquisition was performed on a FACScan cytometer with CELLQuest software (Becton Dickinson) for FSC and SSC parameters, which allowed to distinguish cells with a disrupted membrane from those with an intact one. To exclude debris, an FSC threshold was applied based on the light properties of the cells populations in FSC and SSC modes. Five thousand or 10000 events were collected per sample. Data analysis was performed with CELLQuest software. In some experiments, cells were labelled with PI (1 $\mu \mathrm{g} / \mathrm{ml}$, Sigma) for $10 \mathrm{~min}$ before FACS cytometry.

Measurement of fluorescence possibly revealing production of reactive oxygen species. Possible production of reactive oxygen species was assessed by microscopy and flow cytometry analysis. For each point of analysis, cells were incubated for $15 \mathrm{~min}$ at room temperature, protected from light, with either $2^{\prime}, 7^{\prime}$-DC-FDA (10 $\mu \mathrm{M}$, Sigma-Aldrich) or dihydrorhodamine $123(1 \mu \mathrm{M}$, Sigma-Aldrich). For microscopy, cells in Lab-Tek culture chambers were examined directly after incubation by microscopy using Filter set 09 (Zeiss) for $2^{\prime}, 7^{\prime}$-DC-FDA and Filter set 15 (Zeiss) for dihydrorhodamine 123. For flow cytometry, data acquisition was performed directly after incubation, on a FACScan cytometer with CELLQuest software (Becton Dickinson) using FL1 filter. For acquisitions as a function of time, we used a time resolution of $1 \mathrm{~s}$. Data were analyzed using FlowJo software (Tree Star Inc.). To assess mitochondrial localization of DC-FDA fluorescence, cells were incubated with DIF, DC-FDA $10 \mu \mathrm{M}$ and MitoTracker Orange (Molecular Probes) $500 \mathrm{nM}$ in Lab-Tek chambers for $20 \mathrm{~min}$ and observed under the confocal microscope. Pictures were taken between 20 and $40 \mathrm{~min}$ after DIF addition.

Measurement of ATP Levels. ATP measurements were performed on $1 \mathrm{ml}$ of cell suspension $\left(5 \times 10^{5} \mathrm{cell} / \mathrm{s} / \mathrm{ml}\right)$. Cells were lysed by three sonications of $5 \mathrm{~s}$ (3W, Ultrasonic Processor, Bioblock Scientific) on ice, immediatly followed by the addition of perchloric acid $70 \%(125 \mu \mathrm{l}$ for $1 \mathrm{ml}$ of cell suspension), mixing and centrifugation at $4^{\circ} \mathrm{C}$ for $5 \mathrm{~min}$ at 14000 r.p.m. The supernatant was then collected in a new tube and $\mathrm{pH}$ was adjusted to 7.0 with potassium hydroxide $8 \mathrm{~N}$. After $30 \mathrm{~min}$ on ice and $10 \mathrm{~min}$ centrifugation at $4^{\circ} \mathrm{C}$ at 14000 r.p.m., the supernatant was collected in a new tube. The supernatant could be stored at $-20^{\circ} \mathrm{C}$ for later measurement. ATP levels present in the cellular extract was measured with ATP assay mix (FLAAM, Sigma) by luminometry. Results were expressed as amount of ATP per cell.

Measurement of oxygen consumption. Cells were starved overnight in $10 \mathrm{mM}$ Mes-Na buffer, $\mathrm{pH}$ 6.4. in the presence of cAMP. Aliquots $\left(1 \times 10^{7}\right.$ cells $)$ were then collected by centrifugation, resuspended in $2 \mathrm{ml}$ of Mes-Na buffer and transferred to a thermostated oxygraphic cell fitted with a Clark oxygen electrode. Oxygen consumption was recorded as a function of time.

Incubation with sugars. Given sugars were added to starved cells in LabTek chambers, at the indicated final concentrations, $30 \mathrm{~min}$ before the addition of DIF or of DNP or CCCP. Sugars were: D- + + )-glucose (Merck 1.083770250 or Sigma G 7528; these batches gave the same results), D-(-)-fructose (Sigma F 3510), D- ( + )-galactose (Sigma G 0750), D-( + )-mannose (Fluka 63579), D-sorbitol (Sigma S 3889), sucrose (Sigma S 0389), trehalose (Sigma T 0299), 2-deoxy-Dglucose (Sigma D 6134) and 6-deoxy-D-glucose (Sigma D 9761). After the indicated duration of incubation, cells were checked by microscopy and/or by flow cytometry.

Acknowledgements. We thank INSERM and CNRS for institutional support, and for specific grants Agence Nationale pour la Recherche (DictyDeath ANR-05BLAN-0333-01), the European Community (FP6 STREP TransDeath LSHG-CT2004-511983), the Ministère pour la Recherche (ACl BCMS174), Cancéropôle PACA and Association pour la Recherche contre le Cancer. We thank Jonathan Ewbank, Didier Marguet, Pierre-Henri Puech and Gérard Brandolin for helpful discussions and reading the manuscript.

1. Kerr JFR, Wyllie AH, Currie AR. Apoptosis: a basic biological phenomenon with wideranging implications in tissue kinetics. Br J Cancer 1972; 26: 239-257.

2. Clarke PGH, Clarke S. Historic apoptosis. Nature 1995; 378: 230

3. Golstein P, Aubry L, Levraud JP. Cell-death alternative model organisms: why and which? Nat Rev Mol Cell Biol 2003; 4: 798-807.

4. Lemasters JJ. Dying a thousand deaths: redundant pathways from different organelles to apoptosis and necrosis. Gastroenterology 2005; 129: 351-360.

5. Syntichaki $\mathrm{P}$, Tavernarakis $\mathrm{N}$. The biochemistry of neuronal necrosis: rogue biology? Nat Rev Neurosci 2003; 4: 672-684.

6. Leist M, Single B, Castoldi AF, Kuhnle S, Nicotera P. Intracellular adenosine triphosphate (ATP) concentration: a switch in the decision between apoptosis and necrosis. J Exp Med 1997; 185: 1481-1486.

7. Eguchi Y, Shimizu S, Tsujimoto Y. Intracellular ATP levels determine cell death fate by apoptosis or necrosis. Cancer Res 1997; 57: 1835-1840.

8. Baldauf SL, Roger AJ, Wenk-Siefert I, Doolittle WF. A kingdom-level phylogeny of eukaryotes based on combined protein data. Science 2000; 290: 972-977.

9. Whittingham WF, Raper KB. Non-viability of stalk cells in Dictyostelium. Proc Natl Acad Sci USA 1960; 46: 642-649.

10. Kay RR. Cell differentiation in monolayers and the investigation of slime mold morphogens. Methods Cell Biol 1987; 28: 433-448.

11. Cornillon S, Foa C, Davoust J, Buonavista N, Gross JD, Golstein P. Programmed cell death in Dictyostelium. J Cell Sci 1994; 107: 2691-2704.

12. Levraud JP, Adam M, Luciani MF, de Chastellier C, Blanton RL, Golstein P. Dictyostelium cell death: early emergence and demise of highly polarized paddle cells. J Cell Biol 2003; 160: $1105-1114$ 
13. Otto GP, Wu MY, Kazgan N, Anderson OR, Kessin RH. Macroautophagy is required for multicellular development of the social amoeba Dictyostelium discoideum. J Biol Chem 2003; 278: 17636-17645.

14. Kosta A, Roisin-Bouffay C, Luciani MF, Otto GP, Kessin RH, Golstein P. Autophagy gene disruption reveals a non-vacuolar cell death pathway in Dictyostelium. J Biol Chem 2004; 279: 48404-48409.

15. Halliwell $B$, Whiteman $M$. Measuring reactive species and oxidative damage in vivo and in cell culture: how should you do it and what do the results mean? Br J Pharmacol 2004; 142 231-255.

16. Lieberthal W, Menza SA, Levine JS. Graded ATP depletion can cause necrosis or apoptosis of cultured mouse proximal tubular cells. Am J Physiol 1998; 274 (Part 2): F315-F327.

17. Leist M, Single B, Naumann H, Fava E, Simon B, Kuhnle S et al. Inhibition of mitochondrial ATP generation by nitric oxide switches apoptosis to necrosis. Exp Cell Res 1999; 249 : 396-403.

18. Volbracht $\mathrm{C}$, Leist M, Nicotera P. ATP controls neuronal apoptosis triggered by microtubule breakdown or potassium deprivation. Mol Med 1999; 5: 477-489.

19. Crowe JH, Crowe LM, Oliver AE, Tsvetkova N, Wolkers W, Tablin F. The trehalose myth revisited: introduction to a symposium on stabilization of cells in the dry state. Cryobiology 2001; 43: 89-105.

20. Shaulsky G, Loomis WF. Mitochondrial DNA replication but no nuclear DNA replication during development of Dictyostelium. Proc Natl Acad Sci USA 1995; 92: 5660-5663.

21. Fukuzawa M, Araki T, Adrian I, Williams JG. Tyrosine phosphorylation-independent nuclear translocation of a Dictyostelium STAT in response to DIF signaling. Mol Cell 2001 7: 779-788.

22. Fukuzawa M, Abe T, Williams JG. The Dictyostelium prestalk cell inducer DIF regulates nuclear accumulation of a STAT protein by controlling its rate of export from the nucleus. Development 2003; 130: 797-804.

23. Zhukovskaya NV, Fukuzawa M, Yamada Y, Araki T, Williams JG. The Dictyostelium bZIP transcription factor DimB regulates prestalk-specific gene expression. Development 2006 133: $439-448$.

24. Huang E, Blagg SL, Keller T, Katoh M, Shaulsky G, Thompson CR. bZIP transcription factor interactions regulate DIF responses in Dictyostelium. Development 2006; 133 449-458.

25. Eichinger L, Pachebat JA, Glockner G, Rajandream MA, Sucgang R, Berriman M et al. The genome of the social amoeba Dictyostelium discoideum. Nature 2005; 435: 43-57.
26. Killick KA, Wright BE. Multiple forms of glucokinase from Dictyostelium discoideum. J Bacteriol 1978; 133: 1039-1041

27. Gottlob K, Majewski N, Kennedy S, Kandel E, Robey RB, Hay N. Inhibition of early apoptotic events by Akt/PKB is dependent on the first committed step of glycolysis and mitochondrial hexokinase. Genes Dev 2001; 15: 1406-1418.

28. Pastorino JG, Shulga N, Hoek JB. Mitochondrial binding of hexokinase II inhibits Baxinduced cytochrome $c$ release and apoptosis. J Biol Chem 2002; 277: 7610-7618.

29. Danial NN, Gramm CF, Scorrano L, Zhang CY, Krauss S, Ranger AM et al. BAD and glucokinase reside in a mitochondrial complex that integrates glycolysis and apoptosis. Nature 2003; 424: 952-956

30. Zaid H, Abu-Hamad S, Israelson A, Nathan I, Shoshan-Barmatz V. The voltage-dependent anion channel-1 modulates apoptotic cell death. Cell Death Differ 2005; 12: 751-760.

31. Nutt LK, Margolis SS, Jensen M, Herman CE, Dunphy WG, Rathmell JC et al. Metabolic regulation of oocyte cell death through the CaMKII-mediated phosphorylation of caspase2. Cell 2005; 123: 89-103.

32. Le Goffe C, Vallette G, Charrier L, Candelon T, Bou-Hanna C, Bouhours JF et al. Metabolic control of resistance of human epithelial cells to $\mathrm{H}_{2} \mathrm{O}_{2}$ and $\mathrm{NO}$ stresses. Biochem J 2002; 364 (Part 2): 349-359

33. Richards TA, Cavalier-Smith T. Myosin domain evolution and the primary divergence of eukaryotes. Nature 2005; 436: 1113-1118.

34. Golstein P, Kroemer G. Redundant cell death mechanisms as relics and backups. Cell Death Differ 2005; 12 (Suppl 2): 1490-1496.

35. Otto GP, Wu MY, Kazgan N, Anderson OR, Kessin RH. Dictyostelium macroautophagy mutants vary in the severity of their developmental defects. J Biol Chem 2004; 279: $15621-$ 15629.

36. Chautan C, Chazal G, Cecconi F, Gruss P, Golstein P. Interdigital cell death can occur through a necrotic and caspase-independent pathway. Curr Biol 1999; 9: 967-970.

37. Roisin-Bouffay C, Luciani MF, Klein G, Levraud JP, Adam M, Golstein P. Developmental cell death in Dictyostelium does not require paracaspase. J Biol Chem 2004; 279 11489-11494.

38. Leist M, Jaattela M. Four deaths and a funeral: from caspases to alternative mechanisms. Nat Rev Mol Cell Biol 2001; 2: 589-598.

39. Sussman M. Cultivation and synchronous morphogenesis of Dictyostelium unde controlled experimental conditions. In: Spudich JA (ed). Methods in Cell Biology. Harcourt Brace Jovanovich: New York, 1987, pp. 9-29.

40. Kay RR. Cell differentiation in monolayers and the investigation of slime mold morphogens. In: Spudich JA (ed). Methods in Cell Biology. Ac. Press: Orlando, FL, 1987, pp. 433-448.

\section{Supplementary Information accompanies the paper on Cell Death and Differentiation website (http://www.nature.com/cdd)}

